\title{
Design of a New Intelligent Instrument for Curing Tinnitus
}

\author{
Xianmin Ma \\ College of Electrical and Control Engineering \\ Xi'an University of Science \&Technology \\ Xi'an, 710054, China \\ e-mail:maxm@xust.edu.cn
}

\author{
Zhenzhen Li \\ College of Electrical and Control Engineering \\ Xi'an University of Science \&Technology \\ Xi'an, 710054, China \\ e-mail: zhenzhen408@126.com
}

\begin{abstract}
A novel intelligent instrument design for curing tinnitus is introduced in this paper. According to different extent tinnitus, the many different testing sounds such as square wave, sine wave and natural sounds can be produced and the signal frequency and sound intensity level can be adjusted in order to cure tinnitus disease by this new intelligent instrument. The system is constructed in the upper computer and lower microcontroller C8051F410 as the master chip, the data communication is completed with CAN. Based on the $\mathrm{C}++$ Builder, the interface which can display the data and receive the order is designed so the some information of the tinnitus suffers can be achieved in the upper computer for doctor management. This system has been put into practice and has great application value.
\end{abstract}

Keywords- intelligent instrument, curing tinnitus, C++ Builder, C8051F410

\section{INTRODUCTION}

Sensorineural deafness and nervous tinnitus are the most common tinnitus disease. The concealing therapeutics is recognized as one of the most effective way in the treatment of tinnitus and the effect is significantly better in the medical profession[1]. The traditional tinnitus instrument has many weaknesses such as low ratio of price, instability, niche applications, and low accurate frequency. Tinnitus is one of the most common diseases in China with high incidence. This paper designs a new intelligent instrument for curing tinnitus based on the single chip microcomputer, database technology, and audiology technology. This new digital instrument for curing tinnitus could output pure tone, warble tone, narrowband voice, and natural sound. and the frequency and intensity of sound signal are also adjusted. These sounds directly acting on ear to adjust auditory nerve, so the tinnitus can be disappeared and the hearing is improved. One of the advantages of the instrument is to use CAN technique to detect and cure many patients at the same time.

\section{THE PRINCIPLE OF INTELLIGENT INSTRUMENT FOR CURING TINNITUS}

Based on the former research results an intelligence instrument for curing tinnitus is designed. The patients should consult doctor first. The doctor can refer patients for further tests if it is necessary. The process of detection and treatment in essence send the sound which is characteristic frequency voices and specific sound intensity level to patients. These voices can inhabit compensatory activity of the outer hair cells and relieve the symptom tinnitus. The outer hair cell resides outside the diseases of the cochlea. The sounds of this instrument can be divided into two categories, periodic signal and aperiodic signal. Periodic signal is realized by software programming. Single chip computer (C8051F410) and DDS (Direct Digital Frequency Synthesis) is this technology of main part [2]. The aperiodic signals stored in the large memory of AT45DB161D. The tinnitus diagnosis function diagram is shown in Figure 1.

In Figure1, The upper computer medical system has four parts including inquiry, detection, treatment and statistics. The information sharing between different departments is realized through network database. Lower computer generates various sounds, tone, and loudness according to receiving orders. The communication between host and slave gets through the communication module.

\section{DESIGN OF INTELLIGENT TINNITUS INSTRUMENT}

The instrument hardware mainly includes power modules, single chip microcomputer, power amplifier module, can (Controller Area Network) module. The main function is signal generation, signal extraction, signal attenuation and signal amplifier. The system's hardware devices uses C8051F410 microcontroller as the master chip [3]. Besides, it made use of MCU's interior resource to storage and transfer parameters. It simplified the design of the hardware and software and improved integration. This instrument uses CAN in data communication module [4]. The can bus physical layer interface circuit and single-chip microcomputer directly composes distributed measurement and control system. The hardware system's frame diagram is shown in Figure 2.

In Figure2, The aperiodic signals stored in the large memory of AT45DB161D. The voice signals are passed to frequency power attenuator and power amplifier module. The signal the parents needed outputs by earphone. Attenuator controller decides the combination way of $\mathrm{T}$ attenuator and $\pi$ attenuator.

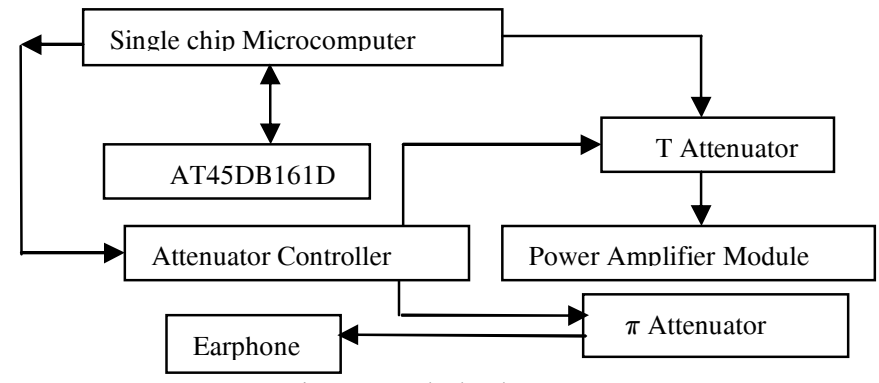

Figure 2. The hardware system 


\section{A. Radio Frequency Power Attenuator}

Radio frequency power attenuator is the key part of this equipment, whose performance determines the accuracy of the test. It includes $\mathrm{T}$ network and $\pi$ network. The attenuation $(1 \mathrm{db}-4 \mathrm{db})$ is obtained by software programming. The final attenuation value is determined by $74 \mathrm{HC} 164$. The instrument has measure up to the standard of the biomedical detection technology. The sound intensity range of this instrument is $-10 \mathrm{db}-120 \mathrm{db}$. The attenuation value is revised by software programming. The attenuation testing values is shown in TABLE I.

\section{B. Power Amplifier}

Signal after $\mathrm{T}$ attenuator does not have the ability of driving load. The power amplifier circuit has been designed in order to improve the signal power. This module is composed of 6 TL084 cascade composition. The TL084 JFET-input (Junction Filed-effect Transistor) operational amplifier family is designed to offer a wider selection than any preciously developed operational amplifier family. The power amplifier is shown in Figure 3.

\section{The Software Design}

The lower computer has seven boards, and their hardware circuit is the same. The only difference is the need of different kinds of sound. This paper takes the right ear of air conduction as example. The flow chart is shown in Figure 4.

\section{The Design of Multiple Interfaces With C++ BUILDER}

Compared with Labwindows/CVI, the greatest advantage for $\mathrm{C}++$ Builder [5] is the direct application of Microsoft ActiveX Data Object (ADO) and SQL Server. It completes with Data Aware Component. Thus, it is more convenient to store and query patient data from database. In this project, the upper computer uses visual programming environment $\mathrm{C}++$ Builder. So this software can perfectly combine full visualization with true object-oriented and the high efficiency and performance of $\mathrm{C}++$. It simplifies the development process without reducing the efficiency of executable code.

The multiple interface form of upper computer mainly include main interface, testing interface, treat interface, print interface, patient query interface, case record interface etc. The upper computer program is firmly in accordance with patient's treatment process. All the patients' data are stored into the database. In our project, we get curve from the Teechart control to analyze patients' testing data. Besides, the QRrport will work together with the database to generate our own statement easily. We apply VFW(Video for Windows) to edit patients photos to obtain Video Capture. AVICap window is used to support real-time video streaming and single frame capture and to control video source. Besides, in auricular treatment the animation is used to observe different voice wave produced by various prescription. The treatment interface in upper computer is shown in Figure 5.

The Intelligent Instrument has been put into operation in a hospital. Various technical indicators have reached the medical requirements. The whole system moves stably and works efficiently, the process is reasonable, and the function is completed. The masking board is shown in Figure 6 .

\section{CONCLUSION}

The intelligent instrument for curing tinnitus has following characters:

(1)With the embedded C8051F410 as a master control chip, the system hardware and software design are simplified, the system integration is improved by the use of internal resources, and the system response is faster.

(2) The output frequency is stable in $30-12000 \mathrm{~Hz}$, so the working range of the intelligent instrument for curing tinnitus is widely enlarged.

(3) The problem of matching different clinical tinnitus is effectively solved, because the treatment prescription is of variety and selectivity.

\section{REFERENCES}

[1] Huang Zhiwu, Chang Wei, Chen Guifang "Tinnitus masking therapy". Journal of Audiology and Speech Pathology, 2004, (6):376 377.

[2] Ni Chenqiang, Ding Yongsheng, "AVR microcontroller signal generator based on DDS technology". Science and Technology Innovation Herald, 2007(32):212

[3] Pan Zhuojin, "C8051F410/1/2/3 Mixed-signal microcontroller databook". NCD, 2006

[4] Fan Hui, "The comparison utility of RS485 and CAN". Shanghai College of Electricity and Machinery Technology, 2005.10:9-11

[5] Liu Guang, "The design and development of $\mathrm{C}++$ Builder database system”. Tsinghua University Press, 2003.11-480 


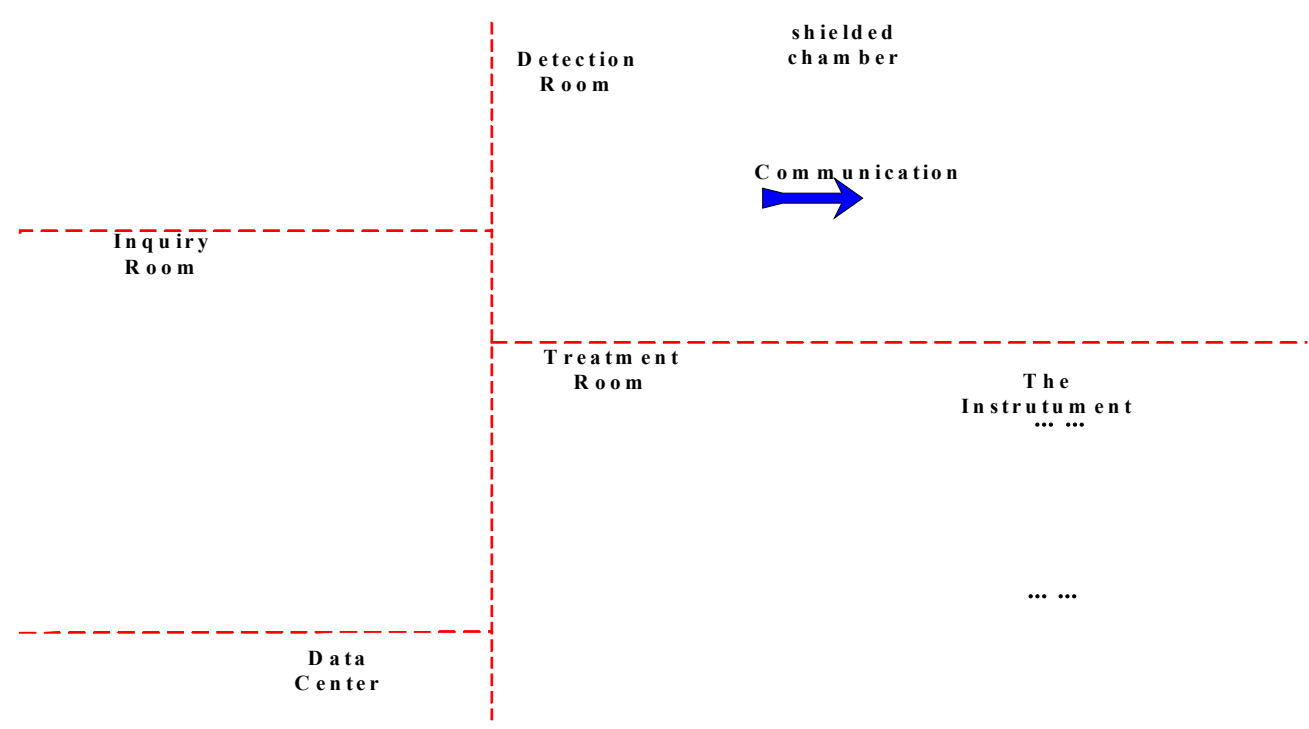

Figure 1. The tinnitus diagnosis function diagram

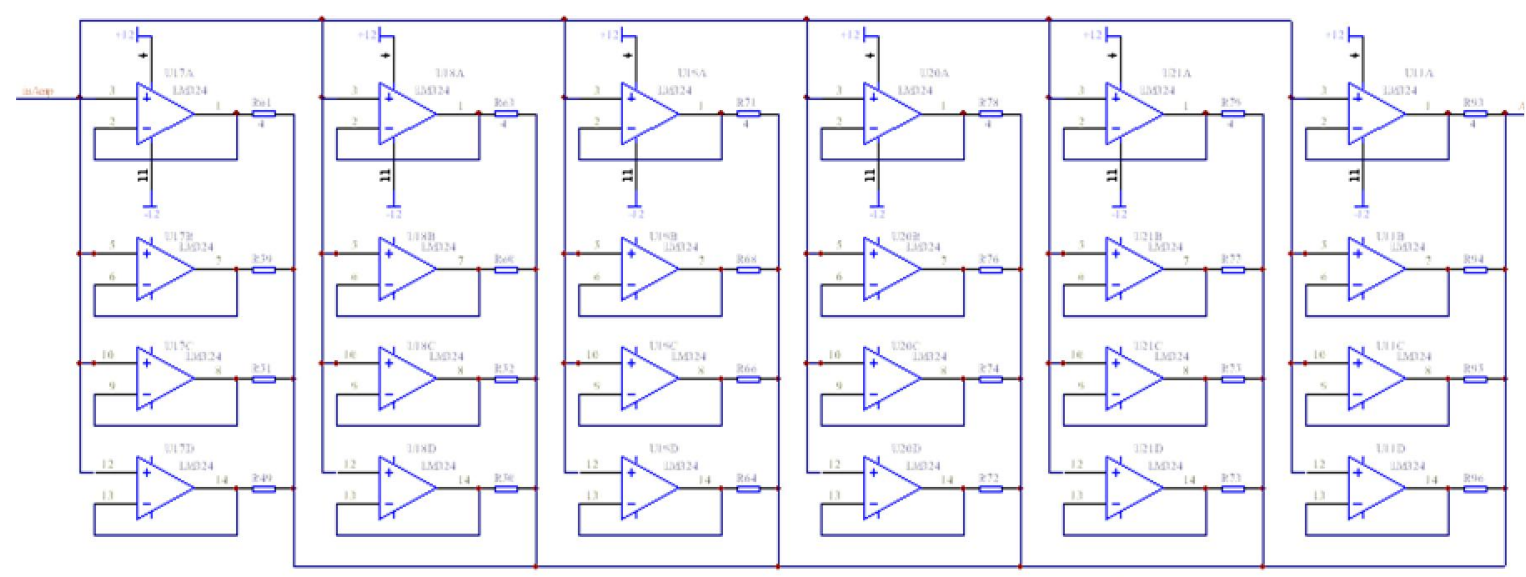

Figure 3. The power amplifier circuit

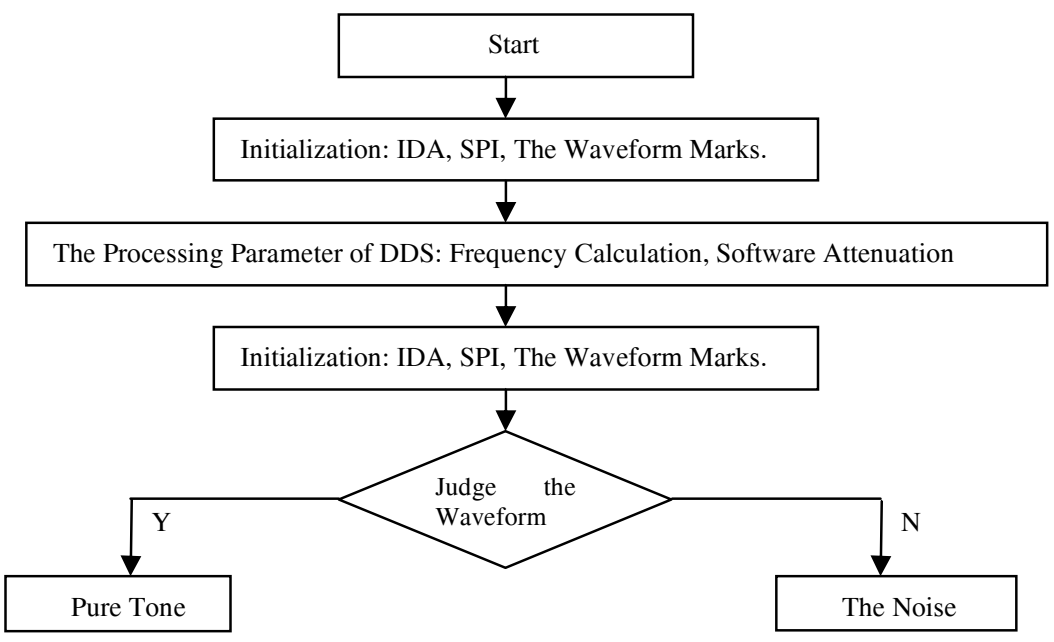

Figure 4. The flow diagram 


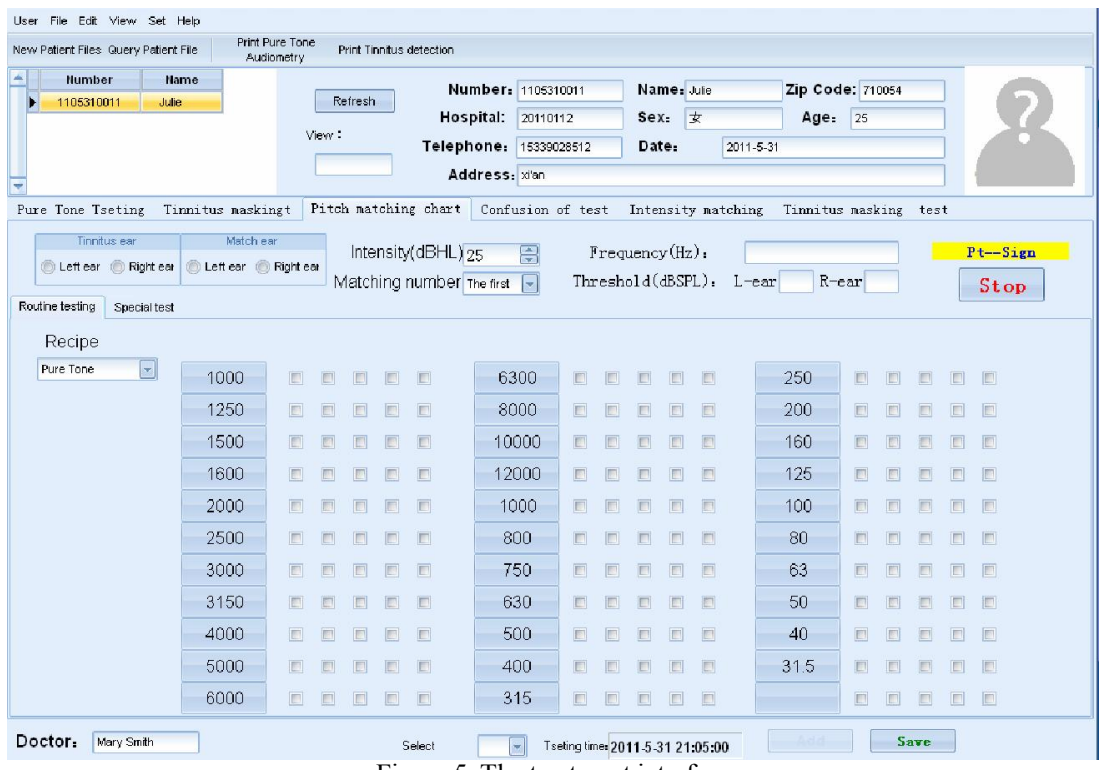

Figure 5. The treatment interface

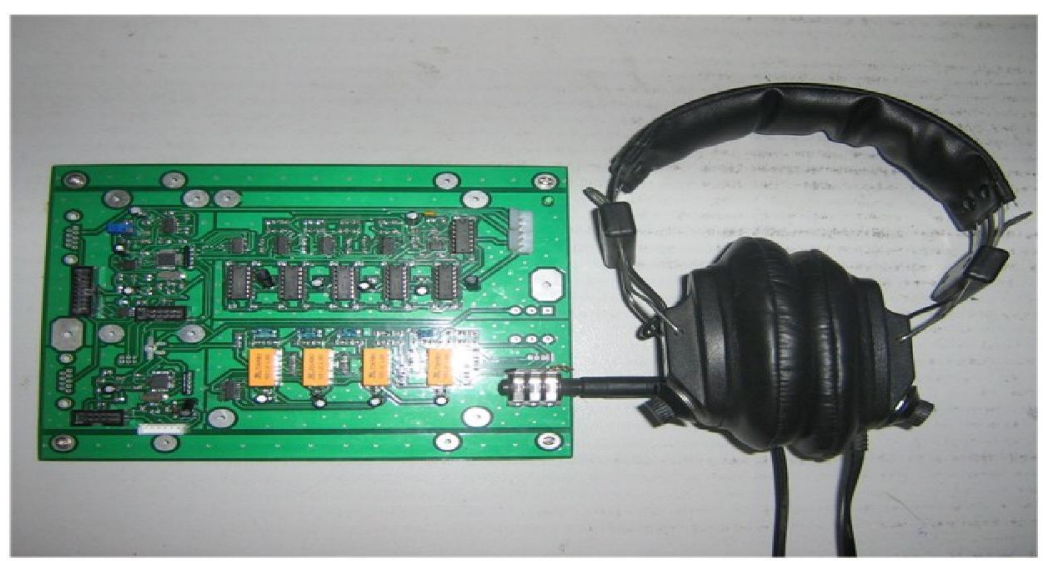

Figure 6. The masking board

TABLE I . The TESTING VALUE OF ATtENUATION

\begin{tabular}{cccccc}
\hline Type & $\begin{array}{c}\text { Attenuation } \\
(d b)\end{array}$ & $\begin{array}{c}\text { Zero } \\
\text { Decrement } \\
(V)\end{array}$ & $\begin{array}{c}\text { Theoretical } \\
\text { Value } \\
(V)\end{array}$ & $\begin{array}{c}\text { Actual } \\
\text { Value } \\
(\text { V) }\end{array}$ & $\begin{array}{c}\text { Correction } \\
\text { Factor }\end{array}$ \\
\hline \multirow{4}{*}{$\mathrm{T}$} & 5 & 5.88 & 3.307 & 3.27 & 1 \\
& 5 & 5.88 & 3.307 & 3.27 & 1 \\
& 10 & 5.88 & 1.859 & 1.86 & 0.96 \\
$\pi$ & 20 & 5.88 & 0.588 & 0.59 & 0.92 \\
& 10 & 5.88 & 1.859 & 1.87 & 0.92 \\
& 20 & 5.88 & 0.588 & 0.586 & 0.92 \\
& 40 & 5.88 & 0.0588 & 0.075 & 0.85 \\
& 40 & 5.88 & 0.0588 & 0.075 & 0.85 \\
\hline
\end{tabular}

